\title{
Experimental Determination of Stress Intensity Factors Due to Residual Stresses
}

\author{
by H.-J. Schindler, W. Cheng and I. Finnie
}

\begin{abstract}
An experimental method is presented that enables stress intensity factors due to residual stress to be determined directly, without prior determination of the residual stress. The method is based on the crack compliance method, where a narrow cut is introduced progressively into the considered component, and the resulting strain change is measured by a strain gage. The required mathematical relations to determine stress intensity factors from strain measurements are established by means of some basic relations of linear elastic fracture mechanics. They are derived explicitly for two exemplary geometrical systems, which allowed for analytical treatment. Experimental data obtained in the case of a steel roller are presented and discussed.
\end{abstract}

\section{Introduction}

Residual stresses can affect the mechanical behavior of a component quite severely, particularly in cases where it is susceptible to subcritical crack growth, like fatigue or stress corrosion cracking. Thus, when performing a safety analysis or a lifetime prediction for such a component, one needs to know the stress intensity factor resulting from residual stress as a function of crack length.

However, the experimental determination of residual stresses as a function of depth below surface is not a trivial task. Traditional methods such as X-ray diffraction combined with layer removal or hole drilling all have limitations. In recent years, the crack compliance method (CC method) as proposed originally ${ }^{1}$ and developed extensively by Cheng and Finnie was successfully applied to several problems of residual stress determination across a full cross section and near the surface. The same basic approach was presented later by $\mathrm{Fett}^{3}$ and Kang. ${ }^{4}$ In short, this method is based on the relaxation of the residual stress by introducing a narrow cut or slit of progressively increasing length and measuring the corresponding strain changes at a suitable location by a strain gage. The advantage of using a narrow cut to relax the residual stress is twofold. First, only a small amount of material is removed, thereby minimizing the risk of causing local plastic deformation and avoiding additional residual stresses. Second, it enables one to apply the well-known theoretical relations of linear elastic fracture mechanics

H. J. Schindler is Senior Research Engineer, Swiss Federal Laboratories for Matcrials Testing and Research, (EMPA), Überlaudstr. 129, CH-8600, Dübendorf, Switzerland. W. Cheng is Assistant Research Engineer, and 1. Fintnic is Professor Emeritus, Department of Mechanical Engineering, University of California, Berkeley, CA 94720.

Original manuscript submitted: February 1996

Final manuscript received: November 15, 1996 to establish the mathematical relations between the original residual stress and the strain change at the location where the strain gage is mounted.

In particular, if the stress intensity factor (SIF) is of interest rather than the residual stress itself, then the $\mathrm{CC}$ method is most effective. It offers, as shown below, the possibility to obtain the SIF directly and precisely without prior knowledge of the residual stress. In fact, it is the principle of the $\mathrm{CC}$ method to calculate the stresses from the SIF rather than vice versa (as it is the case in all other methods).

In the present paper, this aspect of the $\mathrm{CC}$ method is highlighted. First, the general theoretical relations between the unknown stress intensity factor and the measured surface strains are developed. Then, methods of analytical and numerical determination of the relevant functions are shown. Finally, the simplicity and suitability of this experimental technique is demonstrated for the case of a high-strength steel roller.

\section{Strain Change Due to Cutting}

It is the principle of all mechanical methods of residual stress measurement to remove a certain amount of material and to measure the resulting deformation of the body in terms of displacements, stresses or strains. As mentioned in the introduction, the basic idea of the $\mathrm{CC}$ method is to introduce a narrow slit into the considered body along the plane of interest. This causes a redistribution of the residual stress field within the entire body. Thus, the strain change at any arbitrary location in or at the surface of the body due to cutting contains information about the stresses that are released at the cut plane. Knowing the theoretical relationship between these two functions, it is possible to determine the residual stress distribution on the cut plane by the strain measured as a function of cut depth. Because a narrow slit, in an overall consideration of the elastic system, is nearly equivalent to a perfect crack, the well-known equations of linear elastic fracture mechanics can be used to establish the required mathematical relations.

In this section, the general relationship between the SIF of a crack in a two-dimensional residual stress field and the strain change at an arbitrary point on the surface due to crack extension is established. Consider, therefore, an arbitrary body that contains a surface crack of length $a$ and unknown residual stresses (Fig. 1). Now, we assume that the crack is extended by a small increment $\delta a$. At an arbitrary point $M$ on the surface, the change of the surface strain $\delta \varepsilon_{M}$ due to the crack extension can be measured by a strain gage. Following the derivation in Ref. 2 , the strain increment $\delta \varepsilon_{M}$ can be 
expressed by means of the theorem of Castigliano ${ }^{5}$ as

$$
\delta \varepsilon_{M}=\left.\frac{1}{2} \frac{\partial^{2} \delta U}{\partial F \partial s}\right|_{\substack{c=0 \\ s=0}} .
$$

Herein, $\delta U$ denotes the difference in stored elastic energy before and after the crack extension, $F$ is a virtual line force per unit thickness acting tangential on the surface at $M$ and $s$ is the distance between $F$ and $M$. The reaction forces to $F$ can be introduced arbitrarily; often, it is suitable to do it by a second line force acting at the same distance from $M$ at the opposite side of $F$, as shown in Fig. 1. Irwin's ${ }^{6}$ fundamental equation of linear elastic fracture mechanics (LEFM) allows $\delta U$ to be expressed as

$$
\delta U=\frac{B}{E^{\prime}}\left\{\left[K_{I r s}+K_{I F}\right]^{2}+\left[K_{I I r s}+K_{I I F}\right]^{2}\right\} \delta a,
$$

where $K_{I r s}$ and $K_{I I r s}$ denote the SIF due to the residual stress and $K_{I F}$ denotes the SIF due to the virtual force $F, B$ is the thickness of the component (which should be essentially plane) and $E^{\prime}$ is the generalized Young's modulus, which means $E^{\prime}=E$ for plane stress conditions (i.e., thin plate with respect to the distance between the crack tip and $M$ ) and $E^{\prime}=E /\left(1-v^{2}\right)$ for plane strain. Because $K_{I F}$ is a linear function of $F$ and $K_{I r s}$ is independent of $s$, eq (2) inserted in eq (l) can be simplified to

$$
\delta \varepsilon_{M}=\frac{B}{E^{\prime} F}\left[\left.K_{I r s} \frac{\partial K_{I F}}{\partial s}\right|_{s=0}+\left.K_{I / r s} \frac{\partial K_{I I F}}{\partial s}\right|_{s=0}\right] \delta a .
$$

In the following, we restrict ourselves, for the sake of simplicity, to systems in which either $K_{I / F}$ or $K_{I I r s}$ vanish, which is the case either for symmetrical systems with respect to the crack plane or if the forces $F$ do not produce shearing stresses at the plane $y=0$. Replacing the quotient $\delta \varepsilon / \delta a$ by the corresponding derivative, one obtains the desired relation between the strain change at $M$ and the SIF at the incrementally prolonged crack or cut tip to be

$$
K_{\text {Irs }}(a)=\frac{E^{\prime}}{Z(a)} \cdot \frac{d \varepsilon_{M}}{d a},
$$

where

$$
Z(a)=\frac{B}{F}\left(\left.\frac{\partial K_{I F}}{\partial s}\right|_{s=0}\right)
$$

Thus, the SIF of a surface crack in a residual stress field can be experimentally determined by measuring the strain change with respect to cut prolongation $d \varepsilon_{M} / d a$ resulting from progressive cutting-provided that the function $Z(a)$ as introduced in Ref. 7 and defined in eq (4b) is known. $Z(a)$, referred to as the influence function, is a unique function that depends on the component geometry, the cut plane and the measurement point $M$ but not on the residual stress distribution. It characterizes the sensitivity of the measurement point $M$ with respect to the stress intensity factor and the stresses on the cut plane; the bigger the value of $|Z(a)|$, the more sensitive is the measurement.

If the SIF along the entire cross section is to be determined, then it is in general advisable to consider two points

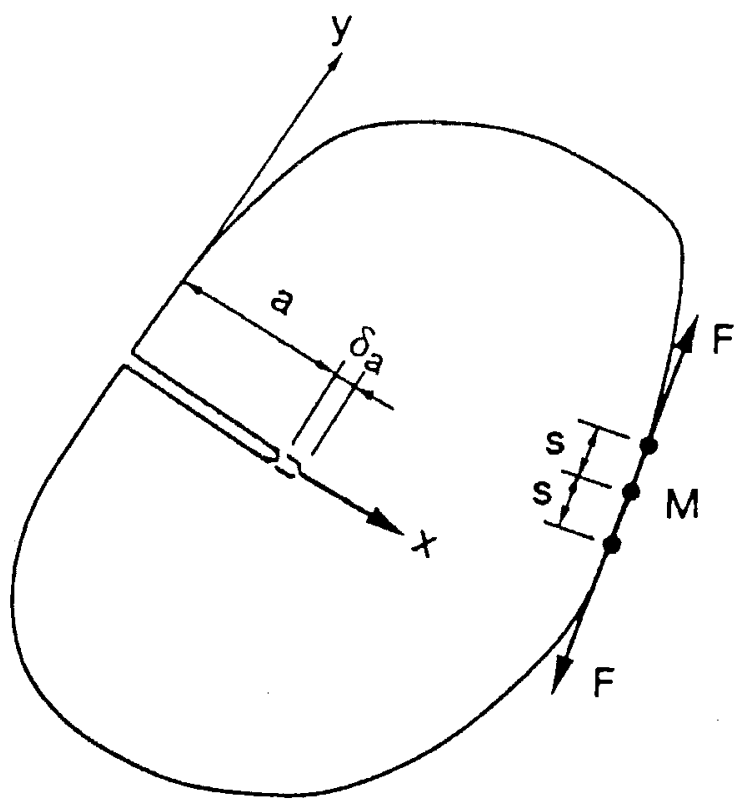

Fig. 1-Mechanical system considered to establish the relationship between the stress intensity factor and the strain at the measurement point $M$

$M$ for strain measurements: one at the front surface, near the crack mouth, and another at the rear surface, at or near its intersection with the cut plane. ${ }^{2}$ The first one is sensitive to the residual stresses in a surface layer with a depth of the same order of magnitude as the distance from the cut mouth (up to about 5 to 10 percent of the cross section), the second is sensitive to the remaining main part of the cross section.

\section{Determination of Influence Functions}

Because the influence function $Z(a)$ is independent of the stress distribution, it needs to be determined only once for a certain geometry. In the following, analytical and numerical methods for its determination are presented. According to eq (4b), $Z(a)$ is essentially given by the derivative of the stress intensity factor of an edge crack due to the line force $F$ with respect to the tangent of the surface at the measurement point $M$. Accordingly, the SIF for this special load case as a function of $s$ has to be determined. In general, the solution cannot be found in handbooks of stress intensity factors. A suitable general technique to obtain $K_{I F}$ is by weight functions as introduced by Bückner. ${ }^{8}$ The stress intensity factor of any crack can be calculated by

$$
K_{I}(a)=\int_{0}^{a} h(x, a) \cdot \sigma_{y}(x) \cdot d x
$$

Herein, $\sigma_{y}(x)$ is the stress distribution acting on the $x$-axis in the uncracked state, and $h(x, a)$ is the weight function, which is a universal function depending on the geometry but not the loading conditions of the considered plane body. It can be determined relatively easily by approximation techniques ${ }^{9-11}$ or, for some cases, can be found in the literature. ${ }^{12}$ Using the weight function technique [eq (5)] to calculate stress intensity factors is particularly suitable when different loading 
conditions, including for instance the residual stress, have to be considered.

Using eq (5), the stress intensity factor due to the virtual line force $F$ is given by

$$
K_{I F}(s)=\int_{0}^{a} h(x, a) \cdot \sigma_{y F}(x, s) \cdot d x
$$

where $\sigma_{y} F(x, s)$ is the distribution of the normal stress along the $x$-axis due to $F$ in the uncracked body. By eq (6), the influence function defined in eq (4b) can be brought to the simple form

$$
Z(a)=\frac{B}{F} \int_{0}^{a} h(x, a) \cdot \frac{\partial \sigma_{y} F}{\partial s}(x, s=0) d x .
$$

Thus, to determine $Z(a)$, one only has to have the stress distribution $\sigma_{y} F(x, s)$ due to the single force $F$ and the weight function $h(x, a)$ where the latter has to be known anyway when dealing with stress intensity factors due to residual stress.

In several cases, as shown in Ref. 13 and by examples in the next section, the desired analytical solutions for $\sigma_{y F}(x, s)$ and their derivative with respect to $s$, respectively, can be obtained relatively easily. However, for many complex geometrical shapes of the component, this will be a difficult task. In these cases, it is recommended to determine $Z(a)$ numerically, e.g., by using the finite element method as follows. The relation (4a) between the stress intensity factor and the strain change at a certain point $M$ holds not only for residual stress fields but for any arbitrary load case of the considered geometrical system. Thus, $Z(a)$ can be obtained directly from eq (4a) by calculating numerically $K_{I}$ and the corresponding strain increment $\Delta \varepsilon_{M}$ at $M$ due to a crack length change $\Delta a$ for an arbitrary loading of the body, referred to as the reference load case, i.e.,

$$
Z(a)=\frac{E^{\prime}}{K_{\text {lref }}(a)} \cdot \frac{\Delta \varepsilon_{M r e f}}{\Delta a}(a) .
$$

$K_{\text {Iref }}$ and $\Delta \varepsilon_{M r e f}$ denote the stress intensity factor and the strain change at $M$, respectively, for the reference load case, which may be chosen to be as simple as possible. Tó reduce the number of calculations of these quantities for different crack lengths, it often is desirable to determine, by curve fitting on the basis of some numerical data, first an algebraic function $\varepsilon_{M r e f}^{*}(a)$ to approximate $\varepsilon_{M r e f}(a)$. Then, $Z(a)$ is obtained by

$$
Z(a)=\frac{E^{\prime}}{K_{\text {Iref }}(a)} \cdot \frac{d \varepsilon_{M r e f}^{*}}{d a}(a) .
$$

\section{Examples}

\section{Short Surface Cut}

When applying the $\mathrm{CC}$ method, the cut is in general introduced starting at the surface of the body. As mentioned above, it is advisable in these cases to choose a first measurement point near the cut at the front surface. As long as the cut length $a$ and the distance $c$ between the strain gage and the cut

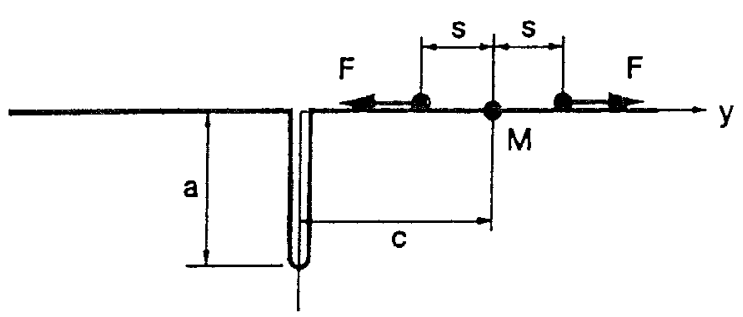

Fig. 2-Mechanical system to determine the influence function for a short surface cut

are small compared to the width $W$ of the cross section, the system can be considered a half-space (Fig. 2). Accordingly, the solution derived subsequently for these conditions are valid for any geometry if $c \ll W$ and $a \ll W$ (which means practically less than 5 to 10 percent of the cross section). Introducing the virtual force $F$ and its reaction of forces acting at distances $\pm s$ from the measurement point $M$, one obtains according to Ref. 5 for the required stress distribution on the cut plane

$$
\begin{aligned}
\sigma_{y F}(x, s) & =\frac{2 F}{\pi B} \cdot\left\{\frac{(c+s)^{3}}{\left[x^{2}+(c+s)^{2}\right]^{2}}\right. \\
& \left.-\frac{(c-s)^{3}}{\left[x^{2}+(c-s)^{2}\right]^{2}}\right\}
\end{aligned}
$$

The weight function $h(x, a)$ for an edge crack in a semiinfinite plate is given in Ref. 12 to be

$$
\begin{aligned}
h(x, a) & =\sqrt{\frac{1-\xi}{2 \pi \cdot a}} \cdot\left[\frac{2}{1-\xi}+0.9788+1.1101 \cdot(1-\xi)\right. \\
& \left.-0.3194 \cdot(1-\xi)^{2}-0.1017 \cdot(1-\xi)^{3}\right]
\end{aligned}
$$

where $\xi=x / a$. Introducing the nondimensional distance $\gamma=c / a$ and inserting eq (10) and eq (9) in eq (7) results in

$$
Z(c / a)=\frac{4}{\pi \cdot a} \cdot \int_{0}^{1} h(\xi) \cdot \gamma^{2} \cdot \frac{3\left(\xi^{2}+\gamma^{2}-4 \gamma^{2}\right.}{\left(\xi^{2}+\gamma^{2}\right)^{3}} d \xi
$$

This influence function is graphically represented in Fig. 3. Note that there is a maximum at about $c / a=4 / 3$, which means that there is maximum sensitivity at the corresponding cut depth. Whereas the sensitivity for shorter cuts is gradually decreasing, it falls steeply for deeper cuts. According to this behavior of the influence function, the optimal location for strain measurements at the front surface is often in a distance of about $0.05 \mathrm{~W}$ (i.e., 5 percent of the cross-section width) from the cut mouth, since the above solution, which strictly holds only for a semi-infinite plate, is restricted to cut depths of about $a<0.1 \mathrm{~W}$ when applied to bodies of finite widths. Influence functions for finite plate widths are given in Ref. 14.

\section{Radial Cut in Circular Disk}

In the case of a disk or solid cylinder, the derivation given in the foregoing section holds only for $a \ll D$ and $s \ll D$, 


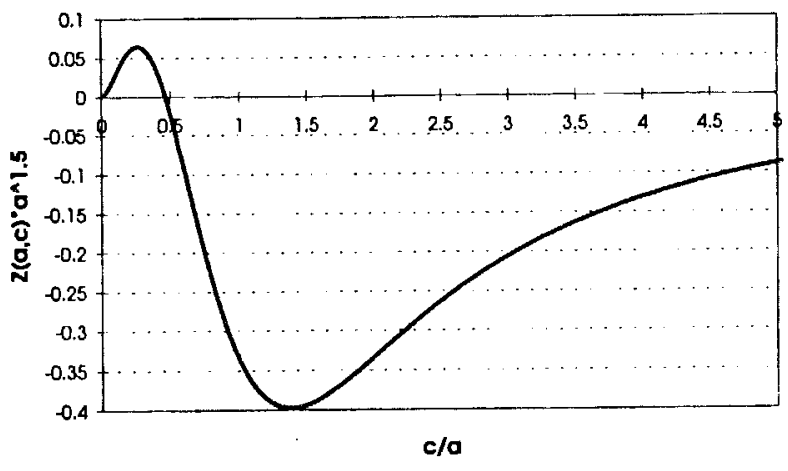

Fig. 3-Influence function $Z$ for a short edge cut as a function of cut depth

where $D$ is denoting the diameter. It has been recognized before ${ }^{2}$ that the remaining 90 to 95 percent of the cross section can be monitored by a strain gage measurement on the rear surface, the most sensitive location being in general at the intersection with the cut plane. Consider, therefore, the system shown in Fig. 4. In order to calculate the strain $\varepsilon_{M}$, a pair of virtual forces $F$ is assumed to act tangentially at points $y= \pm s$, where $s \ll D$ is assumed. $\sigma_{y}(x)$ can be obtained from the general exact solution given in Ref. 5 to be

$$
\sigma_{y F}(x, s)=\frac{2 F}{\pi \cdot B} \cdot\left\{\frac{s^{3}}{\left[(D-x)^{2}+s^{2}\right]^{2}}-\frac{2 s}{D^{2}}\right\} .
$$

Inserting eq (12) into eq (7) leads to

$$
Z(a)=-\frac{4}{\pi \cdot D^{2}} \int_{0}^{a} h(x, a) d x
$$

According to eq (13), $Z(a)$ is proportional to the integral of $h(x, a)$. The same integral appears when the stress intensity for a load case consisting of a homogeneous pressure acting on the crack surface is calculated by the weight function technique. Thus, it is possible to obtain the integral in question from the stress intensity factor of this load case. For the present geometry, an exact solution for the SIF due to a homogeneous pressure $p$ acting on the crack surface is given in Refs. 15 and 16:

$$
K_{I}=1.988 \cdot p \cdot \sqrt{\frac{a}{\left(1-\frac{a}{D}\right)^{3}}} .
$$

The same $K_{l}$ can also be obtained by inserting $\sigma_{y}(x)=p$ in eq (5). Comparing the resulting expression with eq (4) yields

$$
\int_{0}^{a} h(x, a) d x=1.988 \cdot \sqrt{\frac{a}{\left(1-\frac{a}{D}\right)^{3}}} .
$$

Using eq (15) in eq (13) and inserting them into eq (4b) leads to

$$
Z(a)=-\frac{7.952}{\pi \cdot D^{3 / 2}} \cdot \sqrt{\frac{\frac{a}{D}}{\left(1-\frac{a}{D}\right)^{3}}} .
$$

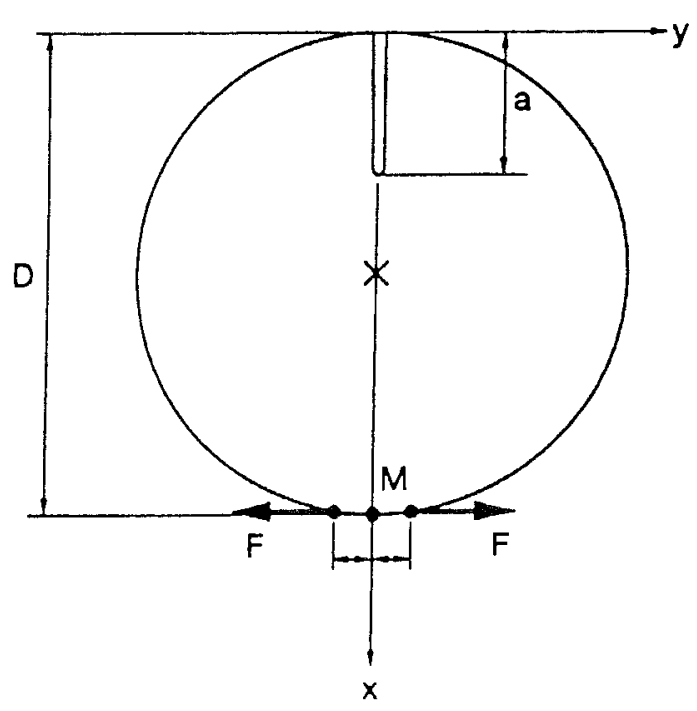

Fig. 4-Circular disk containing a radial edge crack

It is interesting to note that this remarkably simple result is an exact solution; thus, there is no theoretical restriction concerning the crack or cut length. It applies for the whole range of cut depths. However, there are some practical restrictions: for very short cuts (less than about 5 percent of the diameter) the sensitivity is too low, and for very deep cracks, the cut width may be too large compared with the ligament, so the cut can no longer be regarded as an ideal crack.

\section{Experimental Results}

As an example, the residual stress and the corresponding SIF in a roller used in a bridge support is considered. Made of high-strength steel, these rollers exhibited large cracks after some years of, service. ${ }^{17,18}$ Because the service load is predominantly compressive, there was evidence that the residual stresses played an important role in causing this damage. To quantify their influence, a fracture mechanics analysis was performed, ${ }^{17,18}$ which means that the SIF due to the residual stresses has to be determined. For this purpose, an uncracked roller of the same type, which was in service for about 15 years, was used as a representative test specimen. The roller in question had a diameter of $140 \mathrm{~mm}$ and an overall length of about $600 \mathrm{~mm}$. The material is quenched and tempered tool steel.

For facilitating the handling of the test specimen during the experimental procedure, a slice of $6.4 \mathrm{~mm}$ thickness was cut from the roller, forming a disk of $6.4 \mathrm{~mm}$ thickness and $340 \mathrm{~mm}$ diameter. Thereby the axial residual stresses are released, changing the stress state from one of essentially plane strain to one of plane stress, which has to be taken into account in the subsequent fracture mechanics analysis (see discussion in the next session). To avoid additional residual stress, the slicing and the subsequent progressive radial cutting was done by means of an electric discharge wire machine. The width of the cut was about $0.5 \mathrm{~mm}$.

The strain change was measured by strain gages at the two locations indicated by $M 1$ (2.25 $\mathrm{mm}$ from the crack mouth) and $M 2$ (at the opposite side) in Fig. 5. The measured strains 
factor of 1.64 is reported for the case of a water-quenched steel cylinder. Hence, it seems that a factor of 1.46 can be regarded as a best guess, but it can vary probably in a range of about \pm 20 percent.

\section{Physical Significance of the SIF Due to Residual Stresses for the Considered Roller}

According to the discussion above, the values of SIF given in Fig. 7 have to be multiplied by a factor of about 1.43 to characterize the loading state of a crack in the considered steel roller. The plane strain fracture toughness of the highstrength steel that they are made of is about $41 \mathrm{MPa} \sqrt{\mathrm{m}}(1300$ $\mathrm{N} / \mathrm{mm}^{3 / 2}$ ). Thus, considering the SIF due to the residual stresses (Fig. 7), the critical SIF is reached at a crack depth of about $0.05 \mathrm{D}(7 \mathrm{~mm})$. For deeper cracks, the SIF is higher than $K_{l c}$, which means that there is unstable crack growth up to a crack depth of about $a=0.35 D$, where the SIF is falling back to lower values than $K_{I c}$. Thus, crack arrest is likely to occur in this range of crack depths. The subsequent crack growth is subcritical again, with a decreasing growth rate according to the decreasing SIF. As shown in Ref. 17, the contribution of the external load to the SIF is relatively small in the present case, leaving the maximum $K_{l}$ essentially unchanged, but reducing the crack depth of crack arrest from $0.35 D$ to about $0.25 D$ (depending on the crack orientations with respect to the vertical external load. This behavior explains why in the investigated rollers many cracks with depths between $0.3 D$ and $0.5 D$ were found, but hardly any deeper cracks or complete fractures.

\section{Conclusions}

In applications where the stress intensity factors due to the residual stresses are required, the crack compliance method as outlined above is very straightforward, since it enables the SIF to be determined directly without prior determination of the residual stresses, guaranteeing high accuracy and reliability. Experimentaliy, it is very simple: one only needs to introduce a narrow cut along the plane of interest and to measure the strain change due to the cutting at a certain location on the surface of the body. The desired stress intensity factor is proportional to the slope of the measured strain plotted as a function of the depth of the cut. The corresponding proportionality factor is a unique function, the so-called influence function, that depends on the geometry of the considered component and the cut depth. Furthermore, from the determined stress intensity factor as a function of crack depth, it is possible to determine the residual stresses.

The experimental simplicity and straightforwardness of this method to measure stress intensity factors and residual stresses is made possible by a trade-off with special efforts that have to be spent on the theoretical side. Establishing the required theoretical relations between the stress intensity factor and the strain change at the measurement point may require extensive computation. However, since the required influence functions are unique for a given component and cut geometry, this effort has to be spent only once for a certain class of component geometries.

\section{References}

1. Cheng, W. and Finnic, I., "Measurcment of Residual Hoop Stresses in Cylinders Using the Compliance Method," ASME J. Eng. Mat. Tech., 108, 87-92 (1986).

2. Cheng, W. and Finnic, l., "An Overview of the Crack Compliance Method for Residual Stress Mcasurcment," Proc. 4th Int. Conf. Residual Stress, Baltimore, MD, 449-458 (1994).

3. Fett, T., "Bestimmung von Eigenspannungen mittcls bruchmechanischer Bezichungen," Materialprïfung, 29 (4), 92-94 (1987).

4. Kang, K.J., Song, J.H. and Earmme, Y.Y., "A Method for the Mcasure. ment of Residual Stress Using a Fracture Mechanics Approach," J. Strain Analysis, 24, 23-30 (1989).

5. Timoshenko, S.P. and Goodicr, N.P., Theory of Elasticity, 3rd ed., McGraw-Hill, New York (1970).

6. Irwin, G.R., "A nalysis of Stresses and Strains Near the End of a Crack Traversing a Plate," J. Appl. Mech., 24, 361-363 (1957).

7. Schindler, H.J., "Experimentelle Bestimmung von Spannungsintensitätsfaktoren aus Eigenspannungen," Proc. 27th Vortragsveranstaltung des DVM AK Bruchvorgängc, Deutscher Verband für Matcrialforschung und -prïfung c.V., Köln, 421-430 (1995) (in German).

8. Buckner, H., "A Novel Principle for Computation of Stress Intensity Factors," Zcitschrift für angew, Mathematik und Mechanik (ZAMM), 50, 529 (1970).

9. Petrosky, H.J. and Achenbach, J.D., "Computation of the Weight Function from a Stress Intensity Factor," Eng. Fract. Mech., 10, 257-266 (1970).

10. Fett, T. and Munz, D., "Dic Methode der Gewichtsfunktionen zur Berechnung von Spannungsintensitätsfaktoren," Berichtsband der 23, Vortragsveranstaltung des DVM-AK Bruchvorgange, DVM, Berlin, 249-259 (1991) (in German).

11. Schindler, H.-J., "Weight Functions for Decp Cracks and High Stress Gradients," Advances in Fracture Resistance and Structural Integrity, ed. V.V. Panasyuk et al., Pergamon, Oxford, 19.3-205 (1994).

12. Wu, X.R. and Carlsson, A.J., Weight Functions and Stress Intensity Factor Solutions, Pergamon, Oxford (I99]).

13. Schindler, H.-J. and Finnic, I., "Determination of Residual Stresses and the Resulting Stress Intensity Factors in the Ligament of Pre-cracked Components," Proc. 9th lint. Conf. Fract., Sydncy, Advances in Fracture Research, ed. B.L. Karihaaloo et al, Pergamon, Amsterdam, Vol. 1, 523-530 (1997).

14. Schindler, H.J. and Landolt, R., "Experimental Determination of Residual Stress and the Resulting Stress Intensity Factors in Rectangular Plates," 4th European Conf. Residual Stresses (ECRS4), Cluny, France (1996) (to be published in Journal de Physique, colloques series).

15. Gregory, R.D., "A Circular Disc Containing a Radial Edge Crack Opened by Constant Internal Pressure," Mathematical Proceedings of the Cambridge Philosophical Society, 81 497-521 (1977).

16. Gregory, R.D., "The Spinning Circular Disc with a Radial Edge Crack: An Exact Solution," Int. J. Fract., 41, 39-50 (1989).

17. Schindler, H.-J, and Morf, U., "Schadens- und Sicherheitsanalyse an betriebsgeschädigten Stahlrollen von Brückenlagern," Report No. 517, Swiss Federal Office for Roads and Bridges, Bern (1995) (in German).

18. Schindler, H.-J. and Morf, U., "Load Bearing Capacity of Cracked Rollers Containing Residual Stresses," Proc. IOth European Fract., ed. K.H. Schwalbe and C. Berger, Vol. 2, EMAS, 767-774 (1994).

19. Schindler, H.J., "Determination of Residual Stress Distributions from Measured Siress Intensity Factors," Int. J. Fract., 74, R23-R30 (1995).

20. Schindler, H.J., Cheng, W. and Finnic, l., "Measurement of the Residual Stress Distribution in a Disk or Cylinder Using the Crack Compliance Method," Proc, 4th Int. Conf. Residual Stress, Baltimore, MD, 12661274 (1994)

21. Cheng, W., Finnic, 1. and Vardar, 0., "Estimation of Axisymmetric Residual Stress in a Long Cylinder," J. Eng. Mat. Tech., 114, 137-140 (1992). 
factor of 1.64 is reported for the case of a water-quenched steel cylinder. Hence, it seems that a factor of 1.46 can be regarded as a best guess, but it can vary probably in a range of about \pm 20 percent.

\section{Physical Significance of the SIF Due to Residual Stresses for the Considered Roller}

According to the discussion above, the values of SIF given in Fig. 7 have to be multiplied by a factor of about 1.43 to characterize the loading state of a crack in the considered steel roller. The plane strain fracture toughness of the highstrength steel that they are made of is about $41 \mathrm{MPa} \sqrt{ } \mathrm{m}(1300$ $\mathrm{N} / \mathrm{mm}^{3 / 2}$ ). Thus, considering the SIF due to the residual stresses (Fig. 7), the critical SIF is reached at a crack depth of about $0.05 D(7 \mathrm{~mm})$. For deeper cracks, the SIF is higher than $K_{l c}$, which means that there is unstable crack growth up to a crack depth of about $a=0.35 D$, where the SIF is falling back to lower values than $K_{i c}$. Thus, crack arrest is likely to occur in this range of crack depths. The subsequent crack growth is subcritical again, with a decreasing growth rate according to the decreasing SIF. As shown in Ref. 17, the contribution of the external load to the SIF is relatively small in the present case, leaving the maximum $K$, essentially unchanged, but reducing the crack depth of crack arrest from $0.35 D$ to about $0.25 D$ (depending on the crack orientations with respect to the vertical external load. This behavior explains why in the investigated rollers many cracks with depths between $0.3 \mathrm{D}$ and $0.5 \mathrm{D}$ were found, but hardly any deeper cracks or complete fractures.

\section{Conclusions}

In applications where the stress intensity factors due to the residual stresses are required, the crack compliance method as outlined above is very straightforward, since it enables the SIF to be determined directly without prior determination of the residual stresses, guaranteeing high accuracy and reliability. Experimentally, it is very simple: one only needs to introduce a narrow cut along the plane of interest and to measure the strain change due to the cutting at a certain location on the surface of the body. The desired stress intensity factor is proportional to the slope of the measured strain plotted as a function of the depth of the cut. The corresponding proportionality factor is a unique function, the so-called influence function, that depends on the geometry of the considered component and the cut depth. Furthermore, from the determined stress intensity factor as a function of crack depth, it is possible to determine the residual stresses.

The experimental simplicity and straightforwardness of this method to measure stress intensity factors and residual stresses is made possible by a trade-off with special efforts that have to be spent on the theoretical side. Establishing the required theoretical relations between the stress intensity factor and the strain change at the measurement point may require extensive computation. However, since the required influence functions are unique for a given component and cut geometry, this effort has to be spent only once for a certain class of component geometries.

\section{References}

1. Cheng, W. and Finnie, 1., "Measurement of Residual Hoop Stresses in Cylinders Using the Compliance Method," ASME J. Eng. Mat. Tech., 108, 87-92 (1986)

2. Cheng, W. and Finnic, l., "An Overview of the Crack Compliance Method for Residual Stress Measurement," Proc. 4th litt. Conf. Residual Stress, Baltimore, MD, 449-458 (1994).

3. Fett, $T$., "Bestimmung von Eigenspannungen mittels bruchnechanischer Bezichungen," Materialprüfung, 29 (4), 92-94 (1987).

4. Kang, K.J., Song, J.H. and Earmme, Y.Y., "A Method for the Measurement of Residual Stress Using a Fracture Mechanics Approach," J. Strain Analysis, 24, 23-30 (1989).

5. Timoshenko, S.P. and Goodier, N.P., Theory of Elasticity, 3rd ed., McGraw-Hill, Now York (l970).

6. Irwin, G.R., "Analysis of Stresses and Strains Near the End of a Crack Traversing a Plate," J. Appl. Mech., 24, 361-36.3 (1957).

7. Schindler, H.-J., "Experimentelle Bestimmung von Spannungsinten. sitätsfaktoren aus Eigenspannungen," Proc. 27th Vortragsveranstaltung des DVM AK Bruchvorgänge, Deutscher Verband für Materialforschung und -prïfung c.V., Köln, 42I-430 (I995) (in German).

8. Buckner, H., "A Novel Principle for Computation of Stress Intensity Factors," Zeitschrift firr antgew, Mathematik und Mechanik (ZAMM), 50, 529 (I970).

9. Petrosky, H.J. and Achenbach, J.D., "Computation of the Weight Function from a Stress Intensity Factor," Eng. Fract. Mech., 10, 257-266 (1970).

10. Fett, T, and Mutz, D., "Dic Methode der Gewichtsfunktionen zur Bercchnung von Spannungsintensitätsfaktoren," Berichtsband der 23, Vor. tragsveranstaltung des DVM-AK Bruchvorgange, DVM, Berlin, 249-259 (I99I) (in German).

11. Schindler, H.-J., "Weight Functions for Deep Cracks and High Stress Gradients," Advances in Fracture Resistance and Structural Intcgrity, ed. V.V. Panasyuk et al, Pergamon, Oxford, 19.3-205 (1994).

12. Wu, X.R. and Carlsson, A.J., Weight Functions and Stress Intensity Factor Solutions, Pergamon, Oxford (I991).

13. Schindler, H.-J. and Finnic, l., "Determination of Residual Stresses and the Resulting Stress Intensity Factors in the Ligament of Pre-cracked Components," Proc. 9th litt. Conf. Fract., Sydncy, Advances in Fracture Rescarch, ed. B.L. Karihaaloo et al., Pergamon, Amsterdam, Vol. I, 52.3-530 (1997).

14. Schindler, H.J. and Landolt, R., "Experimental Determination of Residual Stress and the Resulting Stress Intensity Factors in Rectangular Plates," 4th European Conf. Residual Stresses (ECRS4), Cluny, France (1996) (to be published in Journal de Physique, colloques series).

15. Gregory, R.D., "A Circular Disc Containing a Radial Edge Crack Opened by Constant Internal Pressure," Mathematical Proceedings of the Cambridge Philosophical Socicty, 81 497-521 (1977).

16. Gregory, R.D., "The Spinning Circular Disc with a Radial Edge Crack: An Exact Solution," Int. J. Fracl., 41, 39-50 (1989).

17. Schindler, H.-J. and Morf, U., "Schadens- und Sicherheitsanalyse an betriebsgeschädigten Stahlrollen von Brückenlagern," Report No. 517, Swiss Federal Office for Roads and Bridges, Bern (I995) (in German).

18. Schindler, H.-J. and Morf, U., "Load Bearing Capacity of Cracked Rollers Containing Residual Stresses," Proc. 10th European Fract., ed. K.H. Schwalbe and C. Berger, Vol. 2, EMAS, 767-774 (1994).

19. Schindler, H.-J., "Determination of Residual Stress Distributions from Measured Stress Intensity Factors," Int. J. Fract., 74, R23-R30 (1995).

20. Schindler, H.J., Cheng, W. and Finnic, I., "Mcasurement of the Residual Stress Distribution in a Disk or Cylinder Using the Crack Compliance Mcthod," Proc. 4th Imt. Conf. Residual Stress, Baltimore, MD, I2661274 (1994).

21. Cheng, W., Finnie, I. and Vardar, 0., "Estimation of Axisymmetric Residual Stress in a Long Cylinder," J. Eng. Mat. Tech., 114, 137-140 (1992). 